\title{
Curious connections: white matter pathways supporting individual differences in epistemic and perceptual curiosity
}

\section{Abbreviated title: Curious connections}

\author{
Ashvanti Valji ${ }^{1}$, Alisa Priemysheva ${ }^{1}$, Carl J. Hodgetts ${ }^{1}$, Alison G. Costigan ${ }^{1}$, \\ Greg D. Parker²,1, Kim S. Graham ${ }^{1}$, Andrew D. Lawrence ${ }^{1}$, Matthias J. Gruber ${ }^{1}$
}

\begin{abstract}
${ }^{1}$ Cardiff University Brain Research Imaging Centre (CUBRIC), School of Psychology, Cardiff University, Cardiff, United Kingdom, CF24 4HQ.

${ }^{2}$ Experimental MRI Centre (EMRIC), School of Bioscience, Cardiff University, Cardiff, United Kingdom, CF10 3AX.
\end{abstract}

Corresponding Authors:

Ashvanti Valji, ValjiA@cardiff.ac.uk;

Matthias J. Gruber, GruberM@cardiff.ac.uk;

Number of pages: 33

Number of Tables: 0

Number of Figures: 5

Number of words for Abstract: 244

Number of words for Introduction: 636

Number of words for Discussion: 1484

Conflict of interests: The authors declare no competing financial interests

Acknowledgements: We would like to thank the funders of this research as well as Ofer Pasternak for providing the free-water corrected pipeline, John Evans and Peter Hobden for scanning support, Samuel Ridgeway and Bethany Coad for support in data collection, Sonya Foley and Marie-Lucie Read for support in data pre-processing. 
2 Across the lifespan, curiosity motivates us to learn, yet curiosity varies strikingly between

3 individuals. Such individual differences have been shown for two distinct dimensions of 4 curiosity: epistemic curiosity (EC), the desire to acquire knowledge about facts, and 5 perceptual curiosity (PC), the desire for sensory information. It is not known, however, 6 whether both dimensions of curiosity depend on different brain networks and whether inter-

7 individual differences in curiosity depend on variation in anatomical connectivity within these 8 networks. Here, we investigated the neuroanatomical connections underpinning individual 9 variation in trait curiosity. Fifty-one female participants underwent a two-shell diffusion MRI 10 sequence and completed questionnaires measuring EC and PC. Using deterministic

11 spherical deconvolution tractography we extracted microstructural metrics (fractional 12 anisotropy (FA) and mean diffusivity (MD)) from two key white matter tracts: the fornix 13 (implicated in novelty processing, exploration, information seeking and episodic memory) 14 and the inferior longitudinal fasciculus (ILF) (implicated in semantic learning and memory). In 15 line with our predictions, we found that EC - but not PC - correlated with ILF microstructure. 16 Fornix microstructure, in contrast, correlated with both EC and PC with posterior 17 hippocampal fornix fibres - associated with posterior hippocampal network connectivity 18 linked to PC specifically. These findings suggest that differences in distinct dimensions of 19 curiosity map systematically onto specific white matter tracts underlying well characterized 20 brain networks. Furthermore, the results pave the way to study the anatomical substrates of 21 inter-individual differences in dimensions of trait curiosity that motivate the learning of distinct forms of knowledge and skills. 


\section{Significance statement}

24 Despite recent interest in curiosity states and the broad spectrum of variation in stable 25 tendencies to experience or express curiosity, the biological correlates of trait curiosity are

26 unknown. Here, we found that specific types of curiosity correlate with microstructure of 27 specific white matter tracts in the brain - the inferior longitudinal fasciculus and the fornix.

28 Our findings on the relationship between specific aspects of curiosity and anatomical 29 connections underlying well characterized brain networks highlight the specificity of trait 30 curiosity. Furthermore, our findings pave the way to further understand inter-individual 31 differences in curiosity and which aspects of curiosity benefit language, memory and other 32 cognitive processes cultivating a deeper knowledge and skill set. 


\section{Introduction}

34 Curiosity is described as the desire for new information that motivates seeking out and acquiring knowledge (Loewenstein, 1994; Litman, 2005). The momentary experience of curiosity can be seen as a motivational state that facilitates knowledge acquisition (Silvia \& Kashdan, 2009; Gottlieb and Oudeyer, 2018). Consistent with this idea, studies have shown that states of high curiosity enhance long-term memory (Kang et al., 2009; Gruber et al., 2014; McGillivray et al., 2015; Marvin and Shohamy, 2016; Stare et al., 2018; Galli et al., 2018). Furthermore, recent neuroimaging evidence suggests that state curiosity enhances memory via increased activation in the mesolimbic dopaminergic circuit including the hippocampus (Gruber et al., 2014; Kang et al., 2009). Notably, the positive effects of state curiosity on memory have been found to greatly vary between individuals in that individual variations observed in the midbrain and hippocampus activity predict the magnitude of memory enhancements (Gruber et al., 2014).

Over the last decades, between-person differences in curiosity as a personality trait (i.e. tendencies to experience and express curiosity) have been well characterized. Based on Berlyne's (1954) suggestion that different types of curiosity are aroused by opportunities for new knowledge or sensory stimulation, trait curiosity has been split into two broad facets: curiosity as engagement with semantic knowledge - epistemic curiosity (EC); or as engagement with sensory stimuli - perceptual curiosity (PC). Building on Loewenstein's (1994) model of aversive curiosity, Litman and colleagues further proposed that these two aspects of curiosity can be further separated into diversive/interest-based and specific/deprivation-based curiosity. Diversive/interest curiosity is linked to positive affect and is thought to energize and direct exploration with the ultimate goal of stimulating one's interest and reduce boredom. In contrast, specific/deprivation curiosity is accompanied by a negative, frustrated feeling of information deprivation and uncertainty, associated with a specific knowledge gap, that people are motivated to eliminate (Berlyne, 1966; Litman, 2005, 2008, Litman and Spielberger, 2003; Litman and Jimerson, 2004). 
The neuroanatomical substrates underpinning individual differences in trait curiosity are unknown. Studies investigating higher-order personality traits subsuming curiosity, however, provide a fruitful starting point to investigate the neuroanatomical connections underlying trait curiosity (DeYoung, 2014; Woo et al., 2014). For example, Privado et al. (2017) found an association between 'openness to experience' and microstructure of the inferior longitudinal fasciculus (ILF), a ventral, temporo-occipital association tract implicated in semantic memory (Herbet et al., 2018; Hodgetts et al., 2015, 2017). Additionally, Cohen et al. (2009) showed that individual differences in novelty seeking were associated with microstructure of the fornix, a key pathway that connects the hippocampus - involved in novelty detection, exploration, information seeking and episodic memory (O'Keefe and Nadel, 1978; Kumaran and Maguire, 2009; Murray et al., 2017; Voss et al., 2017) - to the thalamus, ventral striatum, amygdala and prefrontal cortex (Saunders and Aggleton, 2007; Aggleton et al. 2015).

Here, we used multi-shell diffusion MRI and spherical deconvolution tractography to investigate whether individual differences in ILF and fornix microstructure would be associated with individual differences in trait curiosity. Given the importance of ILF to semantic cognition (Hodgetts et al., 2017; Ripollés et al., 2017; Herbet et al., 2018), we predicted an association between ILF microstructure and EC but not PC. In contrast, given that hippocampal circuitry supports novelty detection, exploratory behaviour and information seeking in many domains (O'Keefe and Nadel, 1978; Kumaran and Maguire, 2009; Murray et al., 2017; Voss et al., 2017) we predicted an association between fornix microstructure and both EC and PC. Further, given evidence of a posterior (fine-grained) to anterior (gistbased) gradient of representational specialization along the long-axis of the hippocampus

83 (Ranganath and Ritchey, 2012; Poppenk et al. 2013; Strange et al., 2014; Murray et al., 84 2017), we predicted that fornical fibres associated with posterior and anterior hippocampus 85 (Christiansen et al., 2017; Saunders and Aggleton, 2007) would be more strongly associated with PC and EC, respectively. 
Materials and Methods

88 Participants

89 Fifty-one healthy female adult undergraduate students, with a mean age of 20 years (SD \pm

901 , range $=19-24)$ participated. They provided written consent prior to participating in the

91 study, which was approved by the Cardiff University Research Ethics Committee, and received a remuneration of approximately $£ 25$ for their participation.

93

$94 \quad$ Trait curiosity measures

95 Participants completed the Epistemic Curiosity Scale (EC) (Litman, 2008) and the Perceptual Curiosity Scale (PC) (Collins et al., 2004), along with other self-report measures not relevant to the current study. The EC scale consists of five interest EC items and five deprivation EC items with participants answering on a scale ranging from 1 (almost never) to 4 (almost always). The interest EC items are associated with behaviours that stimulate positive affect, or involve learning something completely new (e.g. "I enjoy learning about subjects that are unfamiliar to me"). In contrast, deprivation EC items describe behaviours that reduce negative feelings of information deprivation and uncertainty (e.g. "I can spend hours on a single problem because I just can't rest without knowing the answer"). The PC scale (Collins et al., 2004) comprised of twelve items (6 diversive PC items and 6 specific PC items) and again participants respond on a scale that ranged from 1 (almost never) to 4

106 (almost always). The diversive PC items describe exploratory behaviours in which one seeks 107 out new places and a broad range of sensory stimulation (e.g. "I like to discover new places 108 to go"), whereas specific PC describes exploration of novel, specific and sensorially

109 stimulating stimuli (e.g. "When I hear a strange sound, I usually try to find out what caused

110 it"). The Cronbach's alpha coefficients for the scales were all $>=.70$ suggesting good 111 internal consistency. 


\section{Imaging acquisition}

114 Imaging data were obtained at CUBRIC, Cardiff University on a 3 Tesla MRI scanner

115 (Siemens Magnetom Prisma) with a 32-channel head coil. T1-weighted structural 3D images

116 were acquired using an MPRAGE sequence (orientation = sagittal; $T R=2250 \mathrm{~ms}$; $\mathrm{TE}=$

$1173.06 \mathrm{~ms} ; \mathrm{TI}=900 \mathrm{~ms} ;$ flip angle $=9^{\circ} ; \mathrm{FOV}=256 \mathrm{~mm}^{2} ;$ slice thickness $=1 \mathrm{~mm} ;$ voxel size $=$

$1181 \mathrm{~mm}^{3} ;$ number of slices $=224 ;$ bandwidth $=230 \mathrm{~Hz} /$ pixel; total acquisition time $=7$ minutes

11936 seconds).

Diffusion weighted images were acquired using a multi-shell sequence (orientation $=$

121 transversal/axial; $\mathrm{TR}=9400 \mathrm{~ms} ; \mathrm{TE}=67.0 \mathrm{~ms} ; \mathrm{FOV}=256 \mathrm{~mm}^{2}$; slice thickness $=2 \mathrm{~mm}$; voxel

122 size $=2 \mathrm{~mm}^{3}$; number of slices $=80$ ). Diffusion gradients were applied in (i) 30 isotropic

123 directions by using a diffusion-weighted factor $b=1200 \mathrm{sec} / \mathrm{mm}^{2}$, (ii) in 60 isotropic directions

124 by using a diffusion-weighted factor $\mathrm{b}=2400 \mathrm{sec} / \mathrm{mm}^{2}$, and (iii) a volume without diffusion

125 gradients $\left(b=0 \mathrm{sec} / \mathrm{mm}^{2}\right)($ bandwidth $=1954 \mathrm{~Hz} /$ pixel; total acquisition time $=15$ minutes 51

126 seconds).

Diffusion MRI pre-processing

129 T1-weighted structural images were subjected to a 'brain-tissue only' mask using FSL's

130 Brain Extraction Tool (RRID:SCR_002823; Smith, 2002). Using ExploreDTI

131 (RRID:SCR_001643; v4.8.3; Leemans et al., 2009) each b-value image was then co-

132 registered to the T1 structural image. Subsequently, all b-value images were corrected for

133 head motion and eddy currents within ExploreDTI. Tensor fitting was conducted on the b-

1341200 data given the tensor model assumes hindered (Gaussian) diffusion, and at lower b-

135 values more of the signal is due to hindered rather than restricted diffusion (Jones et al.,

136 2013). To correct for voxel-wise partial volume artefacts arising from free water

137 contamination, the two-compartment 'Free Water Elimination' (FWE) procedure was applied

138 to the current $\mathrm{b}-1200$ data - this improves reconstruction of white matter tracts near the 
139 ventricles such as the fornix (Pasternak et al., 2009, 2014), yielding whole brain voxel-wise

140 free-water corrected FA and MD tissue maps. Following FWE, corrected diffusion tensor-

141 derived structural metrics were computed. Fractional anisotropy (FA), reflects the extent to

142 which diffusion within biological tissue is anisotropic (constrained along a single axis). MD

$143\left(10^{-3} \mathrm{~mm}^{2} \mathrm{~s}^{-1}\right)$ reflects overall degree of diffusivity (Vettel et al., 2017). The resulting free

144 water corrected FA and MD maps were inputs for the tractography analysis.

Tractography

147 As higher b-values allow for better fibre orientation estimations (Vettel et al., 2017), we

148 performed tractography on the b-2400 data using damped Richardson-Lucy spherical 149 deconvolution (dRL-SD). Spherical deconvolution provides a direct estimate of the 150 underlying distribution of fibre orientations in the brain and when applied to tractography 151 leads to accurate reconstructions of the major white matter pathway, and an improved ability 152 to describe complex white matter anatomy (Dell'Acqua and Tournier, 2018). The algorithm extracted peaks in the fibre orientation density function (fODF) at the centre of each voxel, where streamlines along the orientation of the fODF peaks were reconstructed using a step size of $0.5 \mathrm{~mm}$. Streamline tracts were terminated if the direction of the pathway changed through an angle greater than $45^{\circ}$ or if the fODF threshold fell below 0.05 .

In ExploreDTI, manual tractography was carried out using AND, NOT, and SEED ROI gates on colour-coded FA maps to extract specific white matter tracts. AND gates (Figure 1 - green) were used to extract fibres that passed through the gate, NOT gates

160 (Figure 1 - red) were used to exclude any fibres that passed through the gate, and finally

161 SEED gates (Figure 1 - blue) were used as a starting point to extract fibres that passed 162 through this gate and then to include only those fibres that then passed through any added 163 AND gates. Manual tractography was carried out on a minimum of 15 subjects in order to 164 calculate a tract model to perform automated tractography on all 51 data sets (Explore DTI; 165 Parker et al., 2013). This procedure enables the construction of white matter tracts in space 
in which streamlines belonging to particular anatomical features of interest consistently

167 project to distinct sub-regions, allowing the reconstruction of streamline data by observing

168 their projected positions (Parker et al., 2013). After running the automated tractography

169 software each tract was visually inspected, and any erroneous fibres were pruned using

170 additional NOT gates. These tract masks from the $b=2400$ data were then intersected with

171 the $b=1200$ free-water corrected FA and MD maps to derive free-water corrected tract-

172 specific measures of FA and MD values for statistical analysis.

174 Inferior Longitudinal Fasciculus tractography. The ILF (Figure 1B) was reconstructed using

175 a two-ROI approach in each hemisphere (Wakana et al., 2007). In the mid-saggital slice of

176 the brain, the coronal crosshair was placed posterior to the corpus callosum. In the coronal

177 plane a SEED gate was drawn around the entire cortex of interest. Next in the coronal view,

178 the last slice where the temporal lobe was separate from the frontal lobe was identified and

179 one AND gate was drawn around the temporal lobe. Any stray fibres not consistent with the

180 ILF pathway were removed with NOT gates. FA and MD of the right and left ILF were summed and averaged to provide a bilateral measure for the main analyses.

183 Fornix tractography. The fornix (Figure 1A) was traced in line with the landmarks described

184 in Catani and Thiebaut de Schotten (2008). In the mid saggital slice of the brain, the coronal crosshair was placed at the anterior commissure and moved approximately 6 voxels posterior in the brain. In the coronal plane, one AND gate was drawn around the fornix bundle where the anterior pillars enter the body of the fornix. Finally, NOT gates were drawn around any protruding areas that were not part of the fornix.

190 Anterior and posterior hippocampal fornix tractography. In addition, we employed a method 191 adapted from prior work to reconstruct the anterior and posterior hippocampal fornix fibres 192 (Christiansen et al., 2017). Both anterior and posterior hippocampal fornix reconstructions 
193 required the AND and NOT gates that were applied during whole fornix tractography. Some

194 NOT gates were augmented to enable better extraction of the anterior and posterior

195 hippocampal streamlines of the fornix. A standard landmark for the anterior-posterior

196 hippocampal boundary was proposed to be a small bundle of grey matter that outlines the

197 most anterior extent of the parahippocampal gyrus that is called uncal apex or uncus

198 (Poppenk et al., 2013). This landmark was identified for each hemisphere separately when

199 carrying out manual tractography of the anterior and posterior hippocampal fornix. In order to

200 perform this, the uncus was first localised at its anterior part and traced to its posterior

201 boundary. The first coronal slice in which the uncus was not visible anymore was used as

202 the landmark in order distinguish between fibres that project into anterior (head of the

203 hippocampus) and posterior hippocampus (body and tail of the hippocampus) (Figure 1C).

After the left and right hemispheric landmarks were identified, one NOT gate on each hemisphere was drawn around the hippocampus to set boundaries for posterior hippocampal fornix tracts, removing fibres that pass through these NOT gates (Figure 1D). After the posterior hippocampal fornix was identified, the same coordinates of the anteriorposterior hippocampal boundary landmark for each hemisphere were used to replace the

209 NOT gates with AND gates for the left and right anterior hippocampal fornix reconstruction

210 (Figure 1E). The posterior, left, and right anterior hippocampal fornix were saved as

211 separate tracts to aid subsequent automated tractography (Figure 2). Note that diffusion

212 tensor metrics of the whole fornix and those averaged across anterior and posterior

213 hippocampal fornix segments were highly correlated $(F A, r(51)=0.940, p<0.001 ; M D, r(51)$

$214=0.942, \mathrm{p}<0.001)$ indicating that the anterior and posterior hippocampal fornix

215 reconstructions matched the whole fornix reconstructions. 

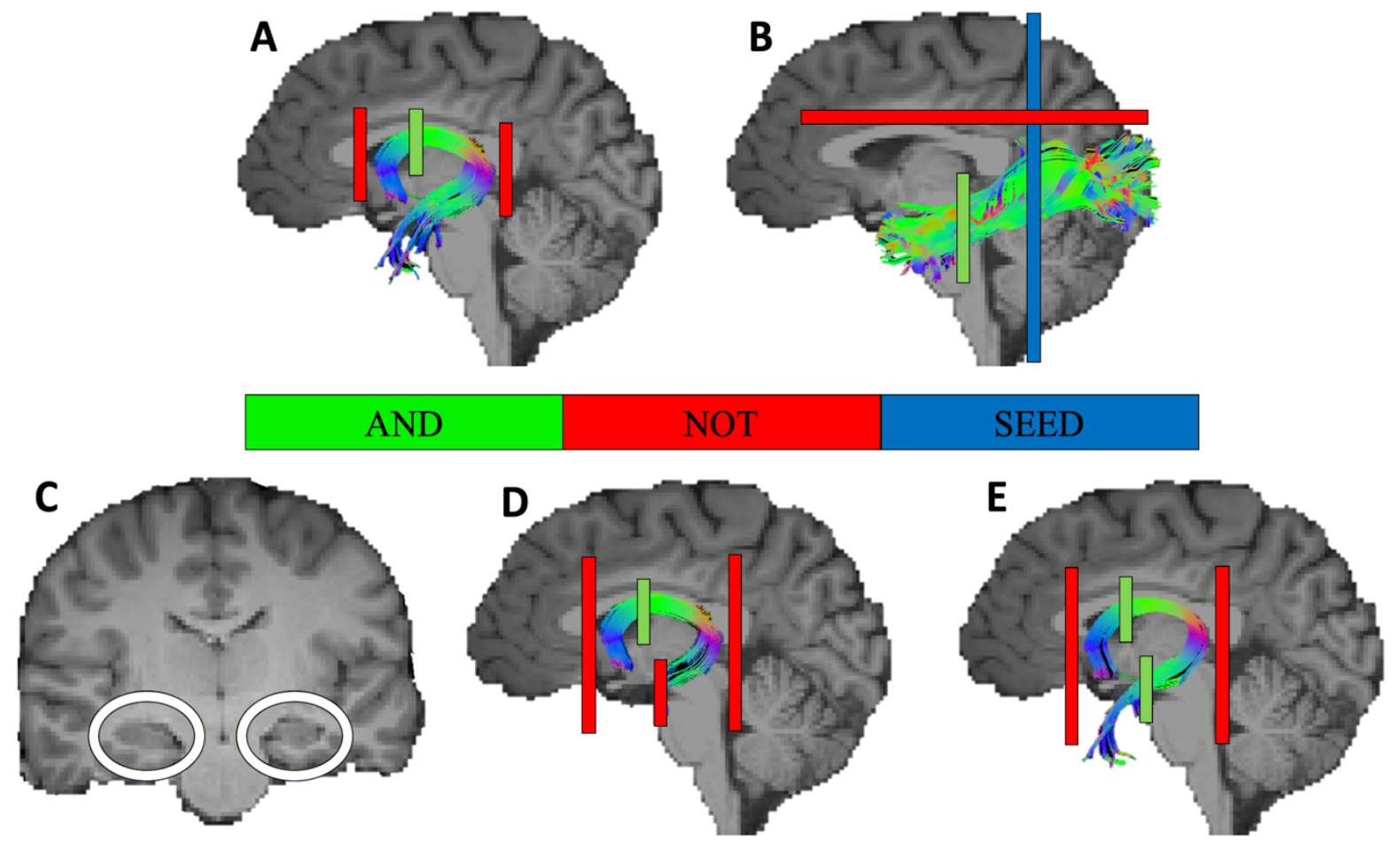

217 Figure 1. Automated tractography reconstructions of the fornix, its anterior and 218 posterior hippocampal fornix fibres and the inferior longitudinal fasciculus (ILF). AND

219 (green), NOT (red), and SEED (blue) ROI gates for each of the tracts are displayed on the sagittal midline plane. (A) Fornix tractography using AND and NOT gates. (B) Left ILF tractography using SEED, AND and NOT gates. (C) Location of AND and NOT gates for tractography of the anterior and posterior hippocampal fornix, respectively.

(D) Posterior

223 hippocampal fornix tractography using one additional NOT gate placed between the head 224 and the body of the hippocampus to only include fornical fibres that connect with posterior 225 hippocampus (i.e., hippocampal body and tail). (E) Anterior hippocampal fornix tractography using one additional AND gate placed between the head and body of the hippocampus (i.e., identical location as NOT gate in (D)) to include fibres that pass through this ROI gate to the anterior hippocampus. 

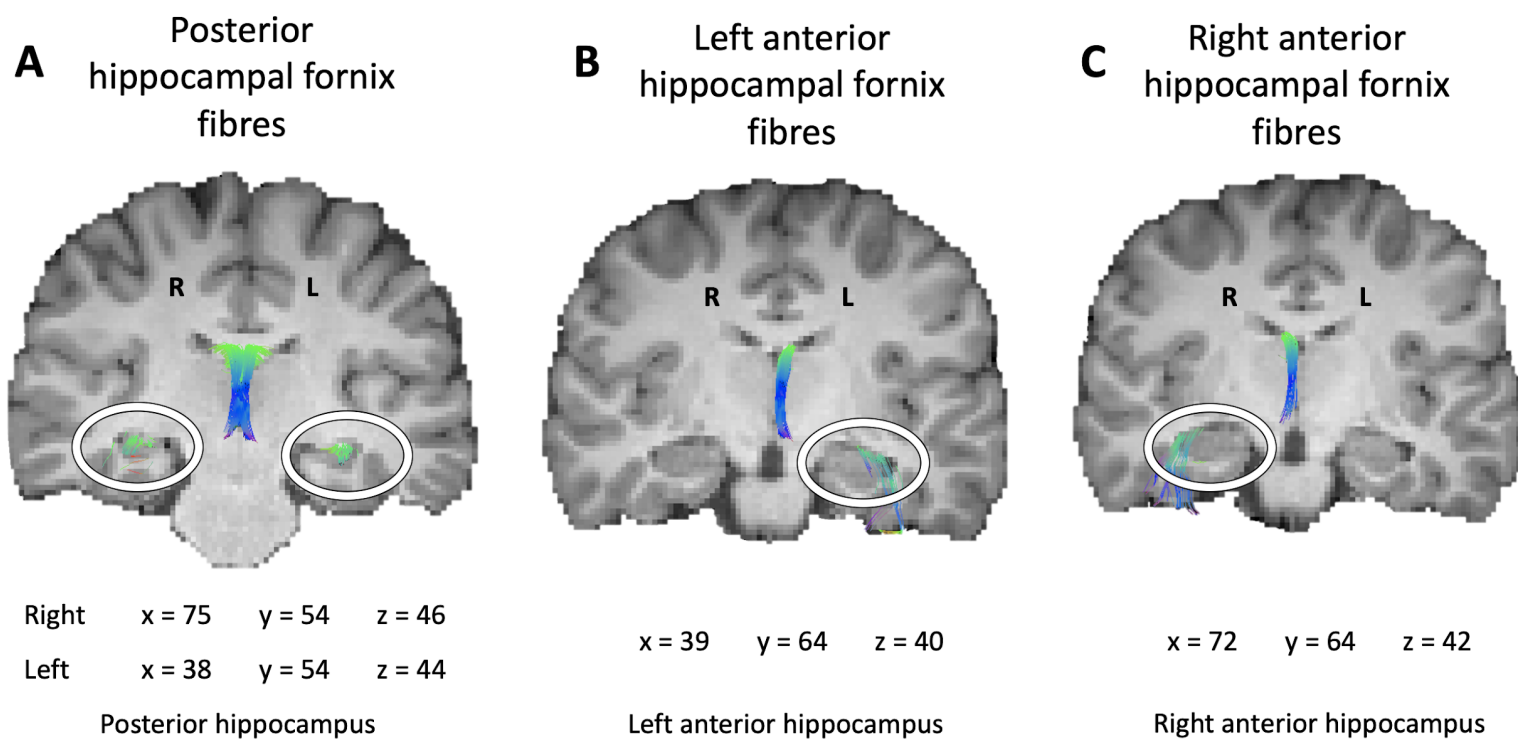

230 Figure 2. Automated tractography reconstructions of anterior and posterior

231 hippocampal fornix fibres on coronal slices. Tractography of the fornix fibres projecting

232 to the posterior hippocampus (A). Tractography of fornix fibres projecting to the left anterior

233 hippocampus (B). Tractography of the fornix fibres projecting to the right anterior

234 hippocampus (C).

Experimental Design and Statistical analyses

237 For the questionnaire data, in the event of missing responses (2 participants failed to give a

238 response to one PC item), the mean value of the remaining items that were answered in the

239 full scale was calculated which then replaced the missing item score. For each curiosity

240 subscale (i.e., the two subscales of PC and EC), we calculated a total score for each

241 participant. Participants' data with diffusion tensor metrics +/- 3SD beyond the group mean

242 were considered as outliers and removed from respective analyses. This resulted in one

243 participant's data being removed from all analyses involving ILF MD and a different

244 participant's data being removed from analyses including bilaterally averaged ILF FA.

To test for associations between curiosity trait scores and microstructure of our 246 selected anatomical tracts, we conducted directional Pearson's correlations using MATLAB 
247 (RRID:SCR_001622). Since higher FA and lower MD is typically associated with higher

248 microstructural integrity (Vettel et al., 2017), we predicted a positive correlation between

249 levels of curiosity and FA and a negative correlation with MD.

To determine whether the Pearson's correlation coefficient $r$ was statistically significant, we performed non-parametric permutation tests that randomly permute the real data between participants. Permutation tests were conducted separately for the two microstructure metrics (i.e., FA and MD) and for the EC and PC scales. Importantly, we corrected for multiple comparisons across the subscales within a curiosity scale (e.g., diversive- and specific PC). The steps were as follows: First, we performed Pearson's correlations on the real data (i.e., correlations between the scores of the two curiosity subscales and the microstructure measure (e.g., diversive PC with ILF MD and specific PC with ILF MD)). Thereby, we obtained the empirical correlation coefficients reflecting the relationship between the two curiosity subscales and a specific microstructure measure. Second, within each curiosity subscale, we shuffled the curiosity scores across participants, which resulted in pairs containing a curiosity score and a microstructure value that is randomly assigned across participants. On these shuffled data, we then calculated surrogate

263 Pearson's coefficients for the two curiosity subscale scores and the microstructure metric,

264 and saved the maximum surrogate Pearson's $r$ across the two correlations (i.e., subscale265 microstructure $\max _{\text {) }}$ (Groppe, Urbach and Kutas, 2011). Third, the second step was repeated 2665000 times. Based on the 5000 permutations, we created a null distribution of all surrogate

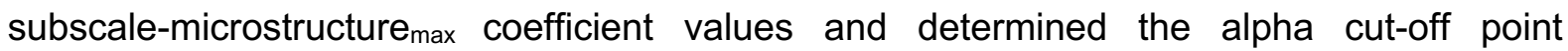
268 ( $p<0.05$; one-sided; i.e., 4750th data point of the surrogate null distribution) in order to test 269 the statistical significance of the real Pearson's coefficients reflecting the relationship 270 between the two subscales and the microstructure measure. This approach allowed us to 271 correct for multiple comparisons across the two subscales within each curiosity scale. In 272 follow-up analyses for specific curiosity subscales (e.g., interest EC subscale), we also 273 performed follow-up permutation tests that corrected for multiple comparisons across both 
274 hemispheres (e.g., left and right ILF MD). The 95\% confidence intervals (CI) for each

275 correlation was derived using a bootstrapping method based on 1000 iterations.

276

277 Results

278 Epistemic curiosity - but not perceptual curiosity - correlates with ILF microstructure

ILF FA. We conducted a series of permutation tests that investigated the relationships between curiosity trait scores and microstructure in a-priori selected anatomical tracts. For each permutation test, we corrected for multiple comparisons for the two subscales separately within the EC and PC scale. The first permutation test targeted ILF FA and EC.

283 We found that bilaterally averaged ILF FA did not significantly correlate with either subscale of EC (deprivation EC, $r(50)=0.143, p_{\text {corr }}=0.243,95 \% \mathrm{Cl}[-0.105,0.364]$; interest $\mathrm{EC}, r(50)$ $\left.=0.191, p_{\text {corr }}=0.151,95 \% \mathrm{Cl}[-0.0734,0.440]\right)$. A further permutation test was conducted on bilaterally averaged ILF FA with the two subscales of PC, where again neither subscale significantly correlated with bilateral ILF FA (specific PC, $r(50)=0.109, p_{\text {corr }}=0.329,95 \% \mathrm{Cl}$ $[-0.229,0.427]$; diversive PC, $\left.r(50)=0.207 ; p_{\text {corr }}=0.122,95 \% \mathrm{Cl}[-0.109,0.453]\right)$.

ILF MD. Targeting ILF MD, a permutation test revealed a significant negative correlation between ILF MD and deprivation EC $\left(r(50)=-0.388, p_{\text {corr }}=0.004,95 \% \mathrm{Cl}[-0.572,-0.124]\right.$, Figure $3 \mathbf{A})$ and a significant negative correlation between ILF MD and interest EC $(r(50)=$ $-0.289, p_{\text {corr }}=0.038,95 \% \mathrm{Cl}[-0.489,0.007]$, Figure 3B). In contrast, bilateral ILF MD was not significantly correlated with any subscale of PC (specific PC, $r(50)=-0.134, p_{\text {corr }}=0.267$, $95 \% \mathrm{Cl}[-0.392,0.157])$; diversive PC $\left(r(50)=0.020, p_{\text {corr }}=0.710,95 \% \mathrm{Cl}[-0.260,0.271]\right)$. 


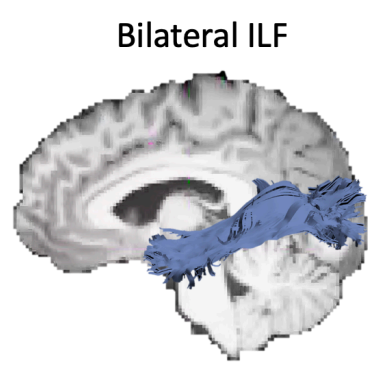

298

300

301

302
A

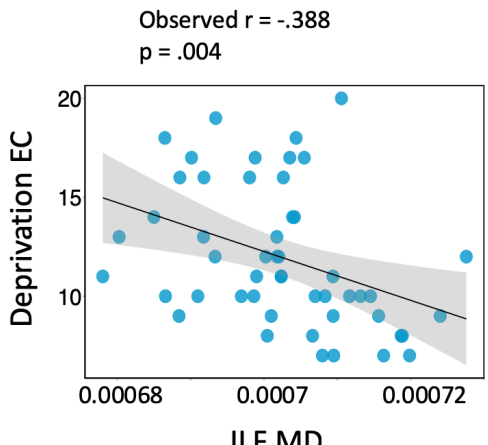

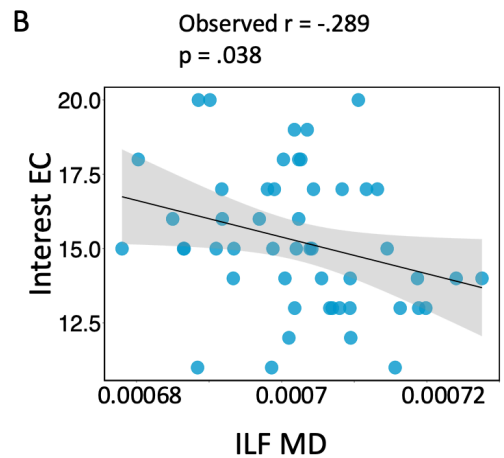

Figure 3. Inferior longitudinal fasciculus microstructure only shows relationship with epistemic curiosity. These results were obtained from non-parametric permutation tests that corrected for multiple comparisons across the two subscales within the Epistemic Curiosity scale (EC). A significant relationship was found between MD of the inferior longitudinal fasciculus (ILF) with deprivation- and interest EC (A, B, respectively). The line of best fit is shown on each scatter plot with 50 data points.

Neuropsychological evidence suggests that semantic knowledge is represented bilaterally in the anterior temporal lobes (ATL) but may show subtle inter-hemispheric (left > right) gradations for verbal stimuli (Rice et al., 2015). Therefore, we asked whether the significant correlation between bilateral ILF MD and both EC subscales were driven specifically by the left as opposed to the right ILF. Separate permutation tests were conducted for each subscale of EC with left ILF MD and right ILF MD as the two variables of interest (i.e., correcting for multiple comparisons across the two hemispheres). The first permutation test on deprivation EC found that both left and right ILF MD significantly correlated with deprivation EC (left ILF: $r(50)=-0.341, p_{\text {corr }}=0.016,95 \% \mathrm{CI}[-0.566,-0.078]$; right ILF: $\left.r(50)=-0.358, p_{\text {corr }}=0.012,95 \% \mathrm{Cl}[-0.564,-0.106]\right)$. The second permutation test investigating whether interest EC correlates with left and right ILF MD indicated a numerical negative relationship for both tracts but neither reached significance with the adopted multiple comparisons correction (left ILF: $r(50)=-0.254, p_{\text {corr }}=0.066,95 \% \mathrm{Cl}[-0.491$, $0.086])$; right ILF: $\left.r(50)=-0.267, p_{\text {corr }}=0.051,95 \% \mathrm{Cl}[-0.472,-0.056]\right)$. 
321 Fornix FA. Regarding fornix FA, permutation tests revealed a significant positive correlation

322 between interest EC and fornix FA $\left(r(51)=0.281, p_{\text {corr }}=0.039,95 \% \mathrm{Cl}[-0.008,0.491]\right.$,

323 Figure 4). In contrast, deprivation EC showed no significant correlation with fornix FA $(r(51)$

$\left.324=0.155, p_{\text {corr }}=0.214,95 \% \mathrm{Cl}[-0.120,0.422]\right)$. A second permutation test was conducted on

325 fornix FA with the two subscales of PC, diversive and specific, but neither subscale

326 significantly correlated with fornix FA (specific PC, $r(51)=0.111, p_{\text {corr }}=0.328,95 \% \mathrm{Cl}$

$327 \quad[-0.266,0.4252]$; diversive PC, $r(51)=0.064, p_{\text {corr }}=0.466,95 \%$ Cl $\left.[-0.204,0.351]\right)$.

329 Fornix MD. Despite the earlier findings of a significant positive correlation between interest

330 EC and fornix FA, permutation tests revealed no significant negative correlation between

331 fornix $\mathrm{MD}$ and interest $\mathrm{EC}\left(r(51)=-0.110, p_{\text {corr }}=0.332,95 \% \mathrm{Cl}[-0.372,0.171]\right)$ or

332 deprivation EC $\left(r(51)=-0.029, p_{\text {corr }}=0.574,95 \% \mathrm{Cl}[-0.314,0.296]\right)$. The second

333 permutation test, investigating the association between fornix MD and the two subscales of

334 PC, also showed that neither specific nor diversive PC significantly correlated with fornix MD

335 (specific PC, $r(51)=-0.250, p_{\text {corr }}=0.070,95 \%$ Cl $[-0.499,0.054]$; diversive $\mathrm{PC},(r(51)=$

Fornix

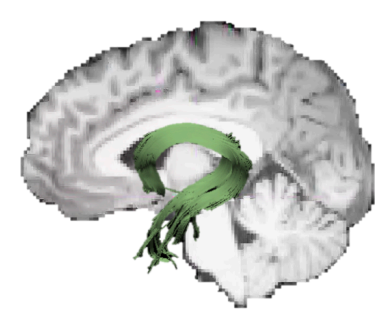

343

Figure 4. Fornix microstructure shows relationship with interest epistemic curiosity.

345 These results obtained from non-parametric permutation tests correcting for multiple 
comparisons across subscales within the Epistemic Curiosity scales (EC). A significant relationship was found between fractional anisotropy (FA) of the whole fornix and interest

348 EC. The line of best fit is shown on the scatterplot with 51 data points.

351 Recent accounts postulate a posterior-anterior gradient of representational granularity along

352 the long axis of the hippocampus, linked to a gradient in anatomical connectivity (Aggleton,

353 2012; Strange et al., 2014), from 'fine' perceptual detail to 'course' or gist-like

354 representations (Poppenk et al., 2013; Robin and Moscovitch, 2017; Sheldon et al., 2019).

355 This account suggests that a correlation might be evident between posterior hippocampal 356 fornix and PC, and anterior hippocampal fornix and EC, respectively. To test this account, 357 we explored the relationship between specific PC (i.e., associated with a detailed perceptual 358 knowledge gap) and anterior/posterior hippocampal fornix MD, given that the correlation 359 between specific PC and whole fornix MD did not reach significance in the preliminary 360 analyses. Conversely, to pinpoint how EC is associated with the anterior/posterior 361 hippocampal fornix FA, we focussed our analyses on interest EC for which we had found a 362 positive correlation with whole fornix FA.

A first permutation test (corrected for multiple comparisons) targeted the three

364 individual fornix segmentations (i.e., left anterior, right anterior, bilateral posterior hippocampal fornix). (Note the anterior hippocampal fornical fibres form the lateral fornix but posterior hippocampal fornical fibres form the medial fornix which cannot easily be separated into separate hemispheres). We found that specific PC significantly correlated

368 with posterior hippocampal fornix MD $\left(r(51)=-0.277, p_{\text {corr }}=0.047,95 \% \mathrm{Cl}[-0.528,0.056]\right.$,

369 Figure 5), but it did not correlate significantly with left or right anterior hippocampal fornix MD (left: $\left(r\left(51=-0.189, p_{\text {corr }}=0.176,95 \% \mathrm{Cl}[-0.451,0.062]\right) ;\right.$ right: $\left(r(51)=-0.028, p_{\text {corr }}=\right.$ 
$3710.610,95 \% \mathrm{Cl}[-0.289,0.264])$. This finding suggests that specific PC might mainly be

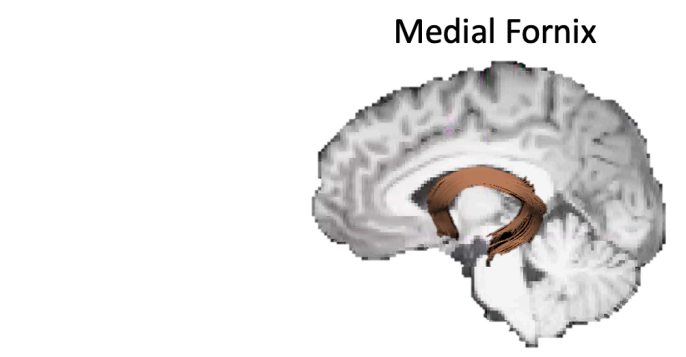

Figure 5. Posterior hippocampal fornix microstructure shows relationship with correcting for multiple comparisons across subscales within the Perceptual Curiosity scales (PC). A significant relationship was found between MD of the posterior hippocampal fornix (i.e., fornix fibres that project specifically into posterior hippocampus) and specific PC. The line of best fit is shown on the scatterplot with 51 data points.

In contrast, although we found that interest EC significantly correlates with whole fornix FA, the three distinct fornix segmentations did not reveal significant correlations with interest EC (left anterior hippocampal fornix FA, $r(51)=0.269, p_{\text {corr }}=0.065,95 \% \mathrm{Cl}[-0.029$, 0.521]; right anterior hippocampal fornix $\mathrm{FA}\left(r(51)=0.080, p_{\text {corr }}=0.479,95 \% \mathrm{Cl}[-0.161\right.$, $0.307]$ ); posterior hippocampal fornix FA, $r(51)=0.272, p_{\text {corr }}=0.062,95 \% \mathrm{Cl}[-0.009$, 0.479]).

In summary, we found that two individual subscales that tap into epistemic and perceptual curiosity traits showed significant correlations with fornix microstructure. In particular, we found that whole fornix FA significantly correlated with interest EC whereas posterior hippocampal fornix microstructure significantly correlated with specific PC. 
398 Curiosity motivates us to seek out information and it facilitates knowledge acquisition

399 (Loewenstein, 1994; Litman, 2005; Silvia and Kashdan, 2009; Gottlieb and Oudeyer, 2018).

400 While a fledgling line of research has shown that curiosity states - the momentary

401 experience of curiosity - enhance hippocampus-dependent memory (for a review, see

402 Gruber et al., 2019), there is also a broad spectrum of variation in stable tendencies to

403 experience or express curiosity. Here, we found that ILF microstructure correlated with both

404 interest and deprivation EC traits, but not with PC traits. Additionally, fornix microstructure was associated with interest - but not deprivation - EC, and specific - but not diversive - PC.

406 In particular, while microstructure of the whole fornix correlated with interest EC, specific PC

407 correlated with the posterior hippocampal fornix. These findings support the notion that

408 curiosity is a multifaceted motivational construct and that distinct aspects of curiosity map

409 onto specific white matter tracts underlying well-characterized brain networks.

\section{Epistemic curiosity and ILF}

411 The ILF, which connects ventral aspects of ATL, occipito-temporal, and occipital cortex 412 (Herbet et al., 2018), appears critical for bidirectional interactions between an ATL-based 413 bilateral semantic 'hub' and representations supported by occipital and middle/posterior 414 temporal regions (Patterson et al., 2007; Lambon Ralph et al., 2017; Chen et al., 2017). In 415 addition to demonstrations of altered ILF microstructure in semantic dementia (Agosta et al., 416 2010), recent studies report associations between ILF microstructure and individual 417 differences in semantic learning (Ripollés et al., 2017) and memory (Hodgetts et al., 2017). 418 Here, we found that participants with reduced diffusivity (i.e., lower MD values) in the ILF 419 showed higher trait scores in both dimensions of EC. Critically, we found that the ILF 420 supported both the general exploration of semantic information motivated by positive affect 421 (EC as a feeling-of-interest) but also the search for specific information in order to close a 422 knowledge gap (EC as an aversive feeling-of-deprivation) (Litman, 2005, 2008; 
423 Loewenstein, 1994; Lauriola et al., 2015). One explanation for this may be that perhaps the

424 more that we learn, the more we are attuned to the gaps in our knowledge, and attending to

425 these gaps is tension-producing and enjoyable at the same time (Loewenstein, 1994). In

426 addition, the association between EC and ILF microstructure is in line with the literature on

427 the higher-order personality trait 'openness to experience', of which curiosity is one facet

428 (Woo et al., 2014). Privado et al. (2017) demonstrated that ILF microstructure was

429 associated with levels of trait 'openness'. Our findings extend this work by pinpointing that

430 the exploration and specific search for semantic information might be one critical factor that

431 carries the association between 'openness' and ILF microstructure.

\section{Curiosity and Fornix}

433 The hippocampus is a medial temporal lobe structure supporting the encoding and recall of 434 long-term memory (Burgess et al., 2002; Davachi, 2006; Eichenbaum et al., 2007; Murray et al., 2018). Given that the hippocampus has been implicated in a number of processes critical to curiosity, including exploration, reward seeking and novelty detection (O'Keefe and Nadel, 1978; Murray et al., 2017; Kumaran and Maguire, 2009; Voss et al 2017), we investigated the relationship between curiosity and the fornix - the principal tract linking the hippocampus with sites beyond the temporal lobe (Postans et al., 2014; Rudebeck et al., 2009). Regarding

440 the relationship between curiosity and fornix microstructure, we performed analyses targeting the microstructure of the whole fornix, but also the anterior and posterior hippocampal fornix segments that correspond to the functional subdivisions of the anterior and posterior hippocampus, respectively (Christiansen et al., 2017; Saunders and Aggleton, 2007). Given current theoretical ideas, the anterior and posterior hippocampal fornix fibres may reflect functional subdivisions of the anterior and posterior hippocampus reflecting gistbased (schematic) and detailed (episodic) information, respectively (Robin and Moscovitch, 2017; Poppenk et al., 2013; Sheldon et al., 2019). Therefore, the present study investigated whether the functional subdivisions of the fornix, connecting to the anterior and posterior hippocampus, may potentially map onto diversive/interest and specific/deprivation curiosity, 
respectively. Consistent with this hypothesis, we found that the posterior hippocampal fornix

451 (but not the anterior hippocampal fornix) showed a relationship with specific PC which is

452 described as the desire to reduce uncertainty by searching for a specific novel perceptual

453 information.

455 In contrast, we found that interest EC positively correlated with microstructure of the whole fornix. Interest EC is described as the desire for diversive exploration and information seeking which is accompanied by positive affect (Litman, 2008). Given these ideas on the

458 functional relevance of interest EC and theoretical ideas and evidence about anterior 459 hippocampal functions, we would have expected that interest EC would show a relationship 460 with anterior hippocampal fornix (i.e., which is strongly connected to anterior hippocampus).

461 The anterior hippocampus has, however, also been proposed to support gist-based and schematic information (Poppenk et al., 2013; Robin and Moscovitch, 2017). Although interest EC reflects the general, explorative search for semantic information, interest EC also triggers search for detailed information rather than gist-based information. Therefore,

465 interest EC might also depend on more posterior hippocampal regions in which detailed 466 'cognitive maps' of the environment, as a means to obtain information, are formed and 467 transition in a graded fashion along the long axis to more gist-based global-contextual 468 'cognitive maps' (Aggleton, 2012; Graham et al., 2010; Murray et al., 2018; Poppenk et al., 469 2013; Strange et al., 2014). In line with this idea of a hippocampal gradient, we found that 470 interest EC did not reach significance for correlations with the left anterior and the posterior 471 hippocampal fornix, but was found to significantly correlate with the whole fornix reflecting 472 the idea that perhaps interest EC depends on fornix fibres that stretch along the whole of the 473 long axis of the hippocampus aligned to EC's dependence on integrative conceptual and 474 detailed maps. 
476 Our study involved questionnaires to tap into distinct curiosity traits. Other recent studies

477 that investigated individual differences in curiosity utilized measures of eye-movement as an objective indicator of visual exploration. For instance, Baranes and colleagues (2015), found curiosity-based enhancement of anticipatory gaze correlated with trait curiosity, and Risko et al. (2012) used a scene-viewing task to demonstrate that participants' PC trait score predicted the degree to which they explored the scenes. These studies using eyemovements to investigate curiosity-based exploration and our present findings on fornix microstructure highlight how individual differences in curiosity play a critical part in the degree of exploration of one's environment.

Limitations and future directions

487 First, our correlation analyses cannot establish causality of one variable over the other. 488 Longitudinal studies would be needed to determine the causality of these relationships to investigate whether trait curiosity shapes white matter connections, vice versa, or whether both reinforce each other in a bidirectional manner. For instance, recent work on adaptive myelination suggests that change in myelination through activity-dependent adaptation of an

492 initially hard-wired process is in response to experiences and contributes to learning 493 (Bechler et al., 2018). Second, interpreting the biological relevance of diffusion metrics from 494 white matter tracts, such as FA and MD, can be challenging. Whilst FA and MD are believed 495 to be inversely related where typically a high FA and low MD suggest greater white matter 496 microstructure (Vettel et al., 2017), we found that for the majority of microstructure-curiosity correlations that only one of the two diffusion metrics significantly correlated with curiosity.

498 These dissociations could be due to the structure of the selected white matter tracts. For 499 example, evidence suggests that FA has been found to be less consistent along a given 500 tract (Yeatman et al., 2012) and other studies also found similar variability in FA and MD 501 measures of the fornix and ILF (e.g., Hodgetts et al., 2017). Inconsistences between FA and 502 MD measures could also be due to a number of biological properties such as axon diameter 
503 and density, myelination and the arrangement of fibres in a given voxel (Beaulieu, 2002). For

504 instance, high FA has been found to reflect high myelin density and structured histological

505 orientation whereas high values of MD is more likely to reflect low myelin density and diffuse

506 histological orientation (Seehaus et al., 2015). This emphasizes the need to use more

507 sophisticated methods in future studies, such as Neurite Orientation Dispersion and Density

508 Imaging, a white matter index which is more informative about brain cellular microstructure

509 than FA and MD alone.

510

511 Conclusion

512 The present study found inter-individual variation in the microstructure of the fornix related to

513 interest EC and inter-individual variation in the microstructure of the ILF related to both

514 interest and deprivation EC. Furthermore, posterior hippocampal fornix microstructure was

515 associated with specific PC. In conclusion, our findings on the relationship between curiosity

516 traits and anatomical connections underlying well characterized brain networks provide a

517 foundation for future studies to examine the relationship between curiosity traits, curiosity

518 states and their neuroanatomical substrates. Our findings pave the way to further

519 understand inter-individual differences in curiosity and which aspects of curiosity benefit

520 language, memory and other cognitive processes cultivating a deeper knowledge and skill

521 set. 


\section{Author contributions}

523 A.V., K.S.G., A.D.L. and M.J.G. contributed to the conception and design of the experiment.

524 A.V. and A.C. contributed to data acquisition. All authors contributed to data analysis and

525 interpretation. A.V. and M.J.G. drafted the manuscript and together with C.J.H., K.S.G. and

526 A.D.L. revised the manuscript. A.D.L. and M.J.G. jointly supervised this work.

\section{$528 \quad$ Funding}

529 This work was supported by a departmental PhD studentship from the School of Psychology

530 at Cardiff University to A.V., a PhD studentship from the Cardiff University Neuroscience and

531 Mental Health Research Institute (NMHRI) to A.C., a Wellcome Strategic Award

$532(104943 / Z / 14 / Z)$ to C.J.H, A.C, K.G., A.D.L., and a COFUND fellowship funded by the Welsh

533 Government and the European Commission and a Sir Henry Dale Fellowship

534 (211201/Z/18/Z) funded by Wellcome and the Royal Society to M.J.G. 


\section{References}

536 Aggleton JP, Wright NF, Rosene, DL, Saunders RC (2015) Complementary patterns of

537 direct amygdala and hippocampal projections to the macaque prefrontal cortex. Cerebral

538 Cortex 25(11):4351-4373.

539 Aggleton JP (2012) Multiple anatomical systems embedded within the primate medial

540 temporal lobe: implications for hippocampal function. Neuroscience \& Biobehavioral

541 Reviews 36(7):1579-1596.

542 Agosta F, Henry RG, Migliaccio R, Neuhaus J, Miller BL, Dronkers NF, Brambata SM, Filippi

543 M, Ogar JM, Wilson SM, Gorno-Tempini ML (2010) Language networks in semantic

544 dementia. Brain 133(1):286-299.

545 Baranes A, Oudeyer PY, Gottlieb J (2015) Eye movements reveal epistemic curiosity in 546 human observers. Vision Research 117:81-90.

547 Beaulieu C (2002) The basis of anisotropic water diffusion in the nervous system - A 548 technical review. NMR in Biomedicine 15(7-8):435-455.

549 Bechler ME, Swire M, ffrench-Constant C (2018) Intrinsic and adaptive myelination-A 550 sequential mechanism for smart wiring in the brain. Developmental neurobiology 78(2):6855179

552 Berlyne DE (1954) A theory of human curiosity. British Journal of Psychology 3: 180-191.

553 Berlyne DE (1966) Curiosity and Exploration. Science 153(3731):25-33.

554 Burgess N, Maguire EA, O'Keefe J (2002) The human hippocampus and spatial and 555 episodic memory. Neuron 35(4):625-641.

556 Catani M, Thiebaut de Schotten M (2008) A diffusion tensor imaging tractography atlas for

557 virtual in vivo dissections. Cortex 44(8):1105-1132.

558 Chen Y, Chen K, Ding J, Zhang Y, Yang Q, Lv Y, Guo Q, Han Z (2017) Brain network for the

559 core deficits of semantic dementia: a neural network connectivity-behavior mapping 560 study. Frontiers in human neuroscience 11:267. 
561 Christiansen K, Metzler-Baddeley C, Parker GD, Muhlert N, Jones DK, Aggleton JP, Vann

562 SD (2017) Topographic separation of fornical fibers associated with the anterior and

563 posterior hippocampus in the human brain: An MRI-diffusion study. Brain and Behavior

$5647(1): 1-8$

565 Cohen MX, Schoene-Bake JC, Elger CE, Weber B (2009) Connectivity-based segregation of

566 the human striatum predicts personality characteristics. Nature Neuroscience 12(1):32-34.

567 Collins RP, Litman JA, Spielberger CD (2004) The measurement of perceptual curiosity.

568 Personality and Individual Differences 36(5):1127-1141.

569 Davachi L (2006) Item, context and relational episodic encoding in humans. Current opinion

570 in neurobiology 16(6):693-700.

571 Dell'Acqua F, Tournier JD (2018) Modelling white matter with spherical deconvolution: How 572 and why?. NMR in Biomedicine 32(4):e3945.

573 DeYoung CG (2014) Openness/Intellect: A dimension of personality reflecting cognitive exploration. In: APA handbook of personality and social psychology: Personality processes and individual differences (Cooper ML, Larsen RJ, eds), pp369-399. Washington, DC:

576 American Psychological Association.

577 Eichenbaum H, Yonelinas AP, Ranganath C (2007) The medial temporal lobe and

578 recognition memory. Annual Review of Neuroscience 30:123-152.

579 Galli G, Sirota M, Gruber MJ, Ivanof BE, Ganesh J, Materassi M, Thorpe A, Loaiza V,

580 Cappelletti M, Craik FIM (2018) Learning facts during aging: the benefits of 581 curiosity. Experimental aging research 44(4):311-328.

582 Gottlieb J, Oudeyer PY (2018) Towards a neuroscience of active sampling and curiosity.

583 Nature Reviews Neuroscience 19:758-770.

584 Graham KS, Barense MD, Lee AC (2010) Going beyond LTM in the MTL: a synthesis of 585 neuropsychological and neuroimaging findings on the role of the medial temporal lobe in 586 memory and perception. Neuropsychologia 48(4):831-853. 
587 Gruber MJ, Gelman BD, Ranganath C (2014) States of Curiosity Modulate Hippocampus-

588 Dependent Learning via the Dopaminergic Circuit. Neuron 84(2):486-496.

589 Gruber MJ, Valji A, Ranganath C (2019) Curiosity and Learning: A Neuroscientific

590 Perspective. In: The Cambridge Handbook of Motivation and Learning. (Renninger KA, Hidi

591 SE, ed), pp 397-417. Cambridge University Press.

592 Herbet G, Zemmoura I, Duffau H (2018) Functional Anatomy of the Inferior Longitudinal

593 Fasciculus: From Historical Reports to Current Hypotheses. Frontiers in

594 neuroanatomy 12:77.

595 Hodgetts CJ, Postans M, Shine JP, Jones DK, Lawrence AD, Graham KS (2015)

596 Dissociable roles of the inferior longitudinal fasciculus and fornix in face and place

597 perception. Elife 4:e07902.

598 Hodgetts CJ, Postans M, Warne N, Varnava A, Lawrence AD, Graham KS (2017) Distinct

599 contributions of the fornix and inferior longitudinal fasciculus to episodic and semantic

600 autobiographical memory. Cortex 94:1-14.

601 Jones DK, Knösche TR, Turner R (2013) White matter integrity, fiber count, and other

602 fallacies: the do's and don'ts of diffusion MRI. Neuroimage 73:239-254.

603 Kang MJ, Hsu M, Krajbich IM, Loewenstein G, McClure SM, Wang JT, Camerer CF (2009)

604 The wick in the candle of leraning. Psychological Science 20(8):963-974.

605 Kumaran D, Maguire EA (2009) Novelty signals: a window into hippocampal information

606 processing. Trends in cognitive sciences 13(2):47-54.

607 Lambon Ralph M, Jefferies E, Patterson K, Rogers TT (2017) The neural and computational

608 bases of semantic cognition. Nature Reviews Neuroscience 18:42-55.

609 Lauriola M, Litman JA, Mussel P, De Santis R, Crowson HM, Hoffman RR (2015) Epistemic

610 curiosity and self-regulation. Personality and Individual Differences 83(1):202-207.

611 Leemans A, Jeurissen B, Sijbers J, Jones DK (2009) ExploreDTI: a graphical toolbox for

612 processing, analyzing, and visualizing diffusion MR data. Proceedings of the 17th Annual

613 Meeting of International Society for Magnetic Resonance in Medicine 17:3536. 
614 Litman JA (2005) Curiosity and the pleasures of learning: Wanting and liking new

615 information. Cognition and Emotion 19:793-814

616 Litman JA (2008) Interest and deprivation factors of epistemic curiosity. Personality and

617 Individual Differences 44(7):1585-1595.

618 Litman JA, Jimerson TL (2004) The measurement of curiosity as a feeling of deprivation.

619 Journal of Personality Assessment 82(2):147-157.

620 Litman J, Spielberger C (2003) Measuring epistemic curiosity and its diversive and specific

621 components. Journal of Personality Assessment 80(1):75-86.

622 Loewenstein G (1994) The psychology of curiosity: A review and reinterpretation.

623 Psychological Bulletin 116(1):75-98.

624 Marvin CB, Shohamy D (2016) Curiosity and reward: Valence predicts choice and

625 information prediction errors enhance learning. Journal of Experimental Psychology: General

626 145(3):266-272.

627 McGillivray S, Murayama K, Castel AD (2015) Thirst for knowledge: The effects of curiosity

628 and interest on memory in younger and older adults. Psychology and Aging 30(4):835-841.

629 Murray EA, Wise SP, Graham KS (2017) The evolution of memory systems: ancestors,

630 anatomy, and adaptations. Oxford University Press.

631 Murray EA, Wise SP, Graham KS (2018) Representational specializations of the

632 hippocampus in phylogenetic perspective. Neuroscience letters 680:4-12.

633 O'Keefe J, Nadel L (1978) The hippocampus as a cognitive map. Oxford: Clarendon Press.

634 Parker GD, Marshall D, Rosin PL, Drage N, Richmond S, Jones DK (2013) Fast and fully

635 automated clustering of whole brain tractography results using shape-space analysis.

636 Proceedings of the International Society for Magnetic Resonance in Medicine. Salt Lake

637 City, USA, 778.

638 Pasternak O, Maier-Hein K, Baumgartner C, Shenton ME, Rathi Y, Westin CF (2014) The 639 estimation of free-water corrected diffusion tensors. In: Visualization and Processing of 640 Tensors and Higher Order Descriptors for Multi-Valued Data (Westin CF, Vilanova A, 
641 Burgeth B, eds), pp. 249-270. Springer, Berlin, Heidelberg.

642 Pasternak O, Sochen N, Gur Y, Intrator N, Assaf Y (2009) Free water elimination and 643 mapping from diffusion MRI. Magnetic Resonance in Medicine 62(3):717-730.

644 Patterson K, Nestor PJ, Rogers TT (2007) Where do you know what you know? The 645 representation of semantic knowledge in the human brain. Nature Reviews 646 Neuroscience 8(12):976-987.

647 Poppenk J, Evensmoen HR, Moscovitch M, Nadel L (2013) Long-axis specialization of the 648 human hippocampus. Trends in cognitive sciences 17(5):230-240.

649 Postans M, Hodgetts CJ, Mundy ME, Jones DK, Lawrence AD, Graham KS (2014) 650 Interindividual variation in fornix microstructure and macrostructure is related to visual 651 discrimination accuracy for scenes but not faces. Journal of Neuroscience 34(36):1212165212126.

653 Privado J, Román FJ, Saénz-Urturi C, Burgaleta M, Colom R (2017) Gray and white matter 654 correlates of the Big Five personality traits. Neuroscience, 349, 174-184.

655 Ranganath C, Ritchey M (2012) Two Cortical Systems for Memory-Guided Behaviour. 656 Nature Reviews. Neuroscience 13(10):713-26.

657 Rice GE, Lambon Ralph MA, Hoffman P (2015) The roles of left versus right anterior 658 temporal lobes in conceptual knowledge: an ALE meta-analysis of 97 functional 659 neuroimaging studies. Cerebral Cortex 25(11):4374-4391.

660 Ripollés P, Biel D, Peñaloza C, Kaufmann J, Marco-Pallarés J, Noesselt T, Rodríguez661 Fornells A (2017) Strength of Temporal White Matter Pathways Predicts Semantic Learning. 662 The Journal of Neuroscience 37(46):1720-17.

663 Risko EF, Anderson NC, Lanthier S, Kingstone A (2012) Curious eyes: Individual differences 664 in personality predict eye movement behavior in scene-viewing. Cognition 122(1):86-90.

665 Robin J, Moscovitch M (2017) Details, gist and schema: hippocampal-neocortical 666 interactions underlying recent and remote episodic and spatial memory. Current Opinion in 667 Behavioral Sciences 17:114-123. 
668 Rudebeck SR, Scholz J, Millington R, Rohenkohl G, Johansen-Berg H, Lee AC (2009)

669 Fornix microstructure correlates with recollection but not familiarity memory. Journal of

$670 \quad$ Neuroscience 29(47):14987-14992.

671 Saunders RC, Aggleton JP (2007) Origin and topography of fibers contributing to the fornix

672 in macaque monkeys. Hippocampus 17(5):396-411.

673 Seehaus A, Roebroeck A, Bastiani M, Fonseca L, Bratzke H, Lori N, Vilanova A, Goebel R,

674 Galuske R (2015) Histological validation of high-resolution DTI in human post mortem

675 tissue. Frontiers in Neuroanatomy 9:98.

676 Sheldon S, Fenerci C, Gurguryan L (2019) A Neurocognitive Perspective on the Forms and

677 Functions of Autobiographical Memory Retrieval. Frontiers in Systems Neuroscience 13:4.

678 Silvia PJ, Kashdan TB (2009) Interesting things and curious people: Exploration and

679 engagement as transient states and enduring strengths. Social and Personality Psychology

680 Compass 3(5):785-797.

681 Smith SM (2002) Fast robust automated brain extraction. Human brain mapping 17(3):143-

682155.

683 Stare CJ, Gruber MJ, Nadel L, Ranganath C, Gómez RL (2018) Curiosity-driven memory

684 enhancement persists over time but does not benefit from post-learning sleep. Cognitive

685 Neuroscience 9:100-115.

686 Strange BA, Witter MP, Lein ES, Moser El (2014) Functional organization of the

687 hippocampal longitudinal axis. Nature Reviews Neuroscience 15(10):655.

688 Vettel JM, Cooper N, Garcia JO, Yeh F-C, Verstynen TD (2017) White Matter Tractography and Diffusion-Weighted Imaging. eLS:1-9.

690 Voss JL, Bridge DJ, Cohen NJ, Walker JA (2017) A closer look at the hippocampus and

691 memory. Trends in cognitive sciences 21(8):577-588.

692 Wakana S, Caprihan A, Panzenboeck MM, Fallon JH, Perry M, Gollub RL, Hua K, Zhang J, 693 Jiang H, Dubey P, Blitz A, van Zijl P, Mori S (2007) Reproducibility of quantitative 694 tractography methods applied to cerebral white matter. Neuroimage 36(3):630-644. 
695 Woo SE, Chernyshenko OS, Longley A, Zhang Z-X, Chiu C-Y, Stark SE (2014) Openness to

696 experience: Its lower level structure, measurement, and cross-cultural equivalence. Journal

697 of Personality Assessment 96(1):29-45.

698 Yeatman JD, Dougherty RF, Myall NJ, Wandell BA, Feldman HM (2012) Tract profiles of 699 white matter properties: automating fiber-tract quantification. PloS one 7(11):e49790. 
Figure 1. Automated tractography reconstructions of the fornix, its anterior and posterior hippocampal fornix fibres and the inferior longitudinal fasciculus (ILF). AND (green), NOT (red), and SEED (blue) ROI gates for each of the tracts are displayed on the sagittal midline plane. (A) Fornix tractography using AND and NOT gates. (B) Left ILF tractography using SEED, AND and NOT gates. (C) Location of AND and NOT gates for tractography of the anterior and posterior hippocampal fornix, respectively.

(D) Posterior hippocampal fornix tractography using one additional NOT gate placed between the head and the body of the hippocampus to only include fornical fibres that connect with posterior hippocampus (i.e., hippocampal body and tail).

(E) Anterior hippocampal fornix tractography using one additional AND gate placed between the head and body of the hippocampus (i.e., identical location as NOT gate in (D)) to include fibres that pass through this ROI gate to the anterior hippocampus.

Figure 2. Automated tractography reconstructions of anterior and posterior hippocampal fornix fibres on coronal slices. Tractography of the fornix fibres projecting to the posterior hippocampus (A). Tractography of fornix fibres projecting to the left anterior hippocampus (C).

Figure 3. Inferior longitudinal fasciculus microstructure only shows relationship with epistemic curiosity. These results were obtained from non-parametric permutation tests

723 that corrected for multiple comparisons across the two subscales within the Epistemic

724 Curiosity scale (EC). A significant relationship was found between MD of the inferior 725 longitudinal fasciculus (ILF) with deprivation- and interest EC (A, B, respectively). The line of best fit is shown on each scatter plot with 50 data points. 
728 Figure 4. Fornix microstructure shows relationship with interest epistemic curiosity.

729 These results obtained from non-parametric permutation tests correcting for multiple

730 comparisons across subscales within the Epistemic Curiosity scales (EC). A significant

731 relationship was found between fractional anisotropy (FA) of the whole fornix and interest

732 EC. The line of best fit is shown on the scatterplot with 51 data points.

733

734 Figure 5. Posterior hippocampal fornix microstructure shows relationship with

735 aspects of perceptual curiosity. These results obtained from non-parametric permutation

736 tests correcting for multiple comparisons across subscales within the Perceptual Curiosity

737 scales (PC). A significant relationship was found between MD of the posterior hippocampal

738 fornix (i.e., fornix fibres that project specifically into posterior hippocampus) and specific PC

739 The line of best fit is shown on the scatterplot with 51 data points. 